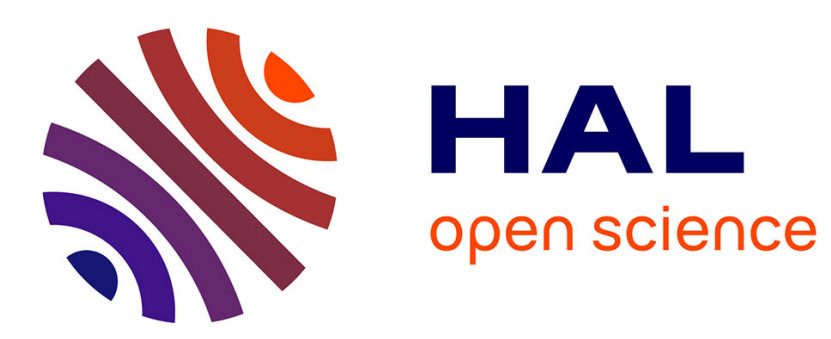

\title{
De l'arrière à l'avant-scène ou de l'intérêt de la réflexivité en sociolinguistique
}

Claudine Moïse

\section{To cite this version:}

Claudine Moïse. De l'arrière à l'avant-scène ou de l'intérêt de la réflexivité en sociolinguistique. Cahiers de sociolinguistique, 2010, Sociolinguistique et réflexivité : vers un paradigme réflexif et herméneutique, 14, pp.171-182. hal-02478673

\section{HAL Id: hal-02478673 \\ https://hal.science/hal-02478673}

Submitted on 19 Feb 2020

HAL is a multi-disciplinary open access archive for the deposit and dissemination of scientific research documents, whether they are published or not. The documents may come from teaching and research institutions in France or abroad, or from public or private research centers.
L'archive ouverte pluridisciplinaire HAL, est destinée au dépôt et à la diffusion de documents scientifiques de niveau recherche, publiés ou non, émanant des établissements d'enseignement et de recherche français ou étrangers, des laboratoires publics ou privés. 


\title{
De l'arrière à lavant-scène ou de lintérêt de la réflexivité en sociolinguistique
}

\author{
Claudine Moïse \\ Université d'Avignon
}

Une énergie dormante, souffrante, séveille à cette complexité, sans avoir peur de se perdre ni de se dénaturer.

Edouard Glissant et Patrick Chamoiseau (2009)

Je souhaite partir du sujet, du sujet chercheur en sciences du langage ; je veux dire cette science du langage que je pratique, l'ethnographie interactionnelle dans une perspective sociolinguistique (Moïse 2007). La réflexivité considérée ici est celle qui amène à questionner les pratiques de la recherche, mais aussi la position du chercheur, pris dans des déterminismes sociaux et des contraintes institutionnelles, forme de psychanalyse de lïnconscient académique (Bourdieu 200I). La réflexivité est, dans un premier temps, un retour sur soi, une exploration de son arrière-scène pour saisir ses propres enchevêtrements, ses motivations et envies de recherche, ses partances et ses désirs de langues. Pourquoi un tel terrain ? Pourquoi telles questions ? Pourquoi ces intuitions ? Quels enjeux professionnels, sociaux, personnels ? Et encore : à quelles fins ? Dans quelles limites ? Ces interrogations, au-delà de la cohérence et de l'harmonie personnelles que l'on y trouve, poussent à interroger notre discipline, forme de réflexivité de la science. Quel en est le bénéfice pour la sociolinguistique ? Quelle sociolinguistique, dans quel paysage en France ?

Le retour sur soi ou, plus exactement la compréhension de nos démarches de recherche, est alors une valeur ajoutée pour notre travail; même si l'exercice est hasardeux, glissant, inépuisable, parce quon risque de s'y perdre, il faut y aller et tenter laventure, parce quill se pose, à un moment donné, en élément nécessaire de notre office.

\section{Les effets d'un décentrement réflexif}

\section{Une recherche problématisée}


Nos histoires et cultures personnelles façonnent pour beaucoup nos champs thématiques et nos perspectives méthodologiques et analytiques; notre " expérience », dans une perspective constructiviste, est toujours là qui détermine notre construction du réel et nos analyses. La confrontation avec mon terrain, dans le nord de l'Ontario au Canada, m’a poussée, pour me sentir à ma place et dans une nécessité de compréhension, à un retour sur moi-même. "L'objet nous désigne plus que nous le désignons " disait déjà Bachelard en I938. Et « l'objet désigne notre façon de composer avec le réel et, au-delà, nous-mêmes, en tant qu’être doué de pensées (Debarbieux 2008 : 63)». Je devais passer de certitudes homogènes et dominantes françaises à une sensibilité hétérogène et minoritaire francophone. La compréhension de nos choix de recherche, par un décentrement constant et intégré dans notre rapport au travail, induit un regard critique sur nos modes d'exploration, de compréhension/interprétation et d'écriture. J'étais partie au Canada, dans le nord de l'Ontario pour entreprendre un doctorat sur la syntaxe du franco-ontarien. Il y eut le choc de la confrontation, d’un paysage inconnu et troublant. J’ai changé de sujet, j’ai voulu réfléchir à «la mise en discours de lidentité minoritaire ". Ce temps d’immersion, lent et long, d’un an a changé mon regard, mais aussi mes modes d’analyse et d'écriture. J'ai eu besoin par la compréhension discursive de raconter des histoires d’identités. Toujours en filigrane de nos démarches de recherche, cette mise à distance est à tricoter et à ne jamais lâcher, parce qu'elle permet tout simplement d'avancer dans les questionnements qui sont les nôtres, de ne jamais abandonner non pas seulement les «comment? " mais surtout les «pourquoi ? " non résolus, et d’en voir les liens et cohérences. Cette mise à distance a donc des effets sur nos questionnements (quelles sont pour moi aujourd'hui les questions sur le monde à travers le langage que jai envie de me poser ?), sur nos façons d’y répondre (quelles méthodes, «chemins à suivre », me semblent les plus adaptées à utiliser ?), sur notre conception de notre objet, la langue par laquelle nous saisissons le social. Le temps a passé et j’ai continué à travailler avec les équipes canadiennes; il y eut quinze années à questionner ce que nous cherchions, les changements linguistiques et culturels des minorités francophones face à la mondialisation, à définir les terrains, à trouver les outils d’analyse les mieux adaptés à notre démarche compréhensive (Moïse, Budach et Kahn 2009). Ces retours, qui nous dédommagent de toute innocence au sujet d’une vérité absolue et objective, touchent toutes les étapes de notre activité scientifique. Ils nous permettent de savoir que nous touchons des bouts de monde et de réalité qui sont les nôtres, contextualisés et historicisés. Cette mise à distance est globale, elle informe, chose bien connue, notre 
rapport aux locuteurs dont nous analysons les paroles, notre place dans le langage, nos représentations et nos façons d'être; elle évite de croire en la transparence des faits en envisageant les enjeux mutuels de pouvoir et d’intérêts, notamment en milieu institutionnel, entre observateurs et observés - ce que Marc Abélès (2004 :35) appelle avec justesse « le sousterrain »-. Sans penser à des instrumentalisations stratégiques, mon activité de recherche, en lien avec le tourisme et l'artistique, a favorisé des mises en réseau entre la France et le Canada, des échanges et des programmations, comme si je participais, dans une mise en abyme vertigineuse, à construire ce que je décrivais. Par une telle élaboration de la réalité à laquelle je coopère, comme actrice moi-même transnationale et mondialisée, il devient central de comprendre mon rôle dans la circulation des représentations et des discours dans le champ social et politique. Je parle pour ma part ici des minorités francophones du Canada, mais on sait les enjeux bien plus âpres des prises de position de certains linguistiques en France, par exemple, sur les questions de la «qualité de la langue française». Nos recherches peuvent servir de légitimation (ou a contrario de repoussoir, donc de contre-légitimation ce qui va, d'une certaine façon, dans le même sens) du politique. Il suffit sans doute de bien le savoir pour «examiner avec auto-réflexion les conséquences de l'action de la recherche " (Heller 2000: 15). Et dans le même temps, cette activité multiple et active, d’analyse et d'action, construit notre domaine disciplinaire, une certaine façon d'être en sociolinguistique, apport modeste mais efficient à la construction de la science.

\section{Une nécessaire altérité}

Le sujet ontologique existe, au-delà de toute pensée humaniste et consensuelle, dans une certitude de son unicité, pris dans l'auto-référence et l'égocentrisme (Morin 1980). La réflexion sur soi, par l'activité de parole, permet de sortir de ce repli. En France, face à des analyses linguistiques largement systémiques, je considère, en contestation, que la langue prise dans une pensée réflexive, n’est plus seulement objet structural désincarné, distancié de soi et des activités sociales et interactionnelles. «Poser la question de la subjectivité c'est simplement rappeler que tout point de vue sur le langage, sur les langues ou sur linteraction, convoque implicitement ou explicitement des points de vue sur le sujet parlant, le rapport à l'autre, le lien social. [...] Tenter de poser la question de la subjectivité, c'est dans le contexte social, historique, épistémologique actuel, faire acte de résistance (Prieur 200I : I3)». La parole, la mienne avec celle de l'autre, par nos catégories de pensée, parle de nous et de nos places 
sociales. Ainsi, l'activité interactionnelle subjectivisée et décentrée nous donne à voir l'autre non pas à partir de nos propres catégories construites et rationalisées mais à partir des siennes propres telles que je peux alors les dé-couvrir. Nos analyses et observations sont des discours non plus seulement sur l'autre mais avec lui. Ce fil d'altérité recompose notre mode d'appréhension de la linguistique, pour quitter une technolinguistique et aller vers une ontolinguistique, toutes deux dans des modèles opposées de démarche, de produit, de conception, de contextualisation notamment (De Robillard 2008: I43). Je rajouterai «d'analyse » dans le sens où les interactions seront au cœur des perceptions des discours et des représentations de l'autre à travers moi-même, dans une attention particulière aux constructions de catégories, aux retours thématiques, aux relances interactionnelles, etc.

Et ce va-et-vient d’altérité nous pose nous-mêmes comme sujet, dans une découverte de notre différence à l’autre. Le terrain, le réel à observer, constitué d’altérité, nous construit autant que nous le construisons comme objet de recherche, si nous acceptons de renoncer à l'aplomb (aux certitudes d'une place légitimée) et au surplomb (aux certitudes du savoir acquis), tâche facilitée quand se fait, par une activité réflexive, la cohérence avec soi-même en tant que non seulement sujet de recherche, mais sujet de vie, rassemblé dans une totalité de soi, mobilisé dans toutes ses subjectivités. Saisir le réel passe alors par une rencontre d'altérité en langue qui s'opère nécessairement dans un rassemblement de soi à soi. Travail, qui permet contre toute tradition positiviste et objectivante, une appréhension plus juste de la complexité de la réalité et des phénomènes que nous étudions, quand nous nous considérons comme partie prenante du monde que nous observons. Mais encore faut-il sortir du paradigme entendu, celui d'une science qui serait plus juste qu’elle serait coupée de celui qui la fait. Les années ont passé et Tzvetan Todorov, chantre du surréalisme, revient sur la coupure entre le sujet de science et le sujet de vie : « je me suis rendu compte que je voulais à la fois nourrir, autant que possible, ma pensée et mon expérience, et être prêt à vivre selon les conclusions auxquelles je pouvais être amené par le raisonnement. [...] Mais rien de ce que jarrivais à penser sur le langage ou la littérature n'avait de relation avec mes convictions ou sympathies, telles que je les éprouvais dans les heures qui n'étaient pas consacrées au travail. Plus même : la logique de ces sciences semblait exclure a priori toute interférence de ce genre, puisque le travail était réputé d’autant mieux fait qu’il était plus « objectif », c'est-à-dire quỉl avait permis d'effacer toute trace du sujet que j’étais ou des jugements de valeur que je pouvais porter (Todorov I989 : 9-IO)». 


\section{La prise en compte de la complexité}

La réflexivité fait sentir lỉncontournable complexité; complexité non feinte de nous-mêmes, de lautre et du monde dans une conscience du désordre, quill soit physique ou social, comme principe productif d'ordre et de compréhension (Morin 1977, 1980). L'exercice revient à accepter, avec toutes les difficultés possibles, que notre recherche est faite de hasards, d'imprévus, de changements, de zones d'ombre, qu'elle ne peut toujours être maitrisée, que les résultats (et notre écriture) seront de l'après-coup, eux-mêmes réorganisés selon quelque modèle théorique ordonné. J'ai dû à un moment essayer de comprendre la construction discursive du Nouvel Ontario (Moïse 2004), de ce territoire en partie francophone qui a construit sa francité. Il m’a fallu du temps et des vues obliques pour en saisir les différents discours hégémoniques historicisés, faits quils étaient de continuité et de rupture, de divergences et dimbrications. Une première analyse énonciative montrait la place singulière en je du sujet dans un discours mondialisé qui supplantait la force d’un nous plus communautaire d'un discours militant et modernisant des années 70 . Mais c'était insuffisant ; ce sont, entre autres, le croisement des positions des acteurs et de leurs intérêts, la compréhension du mythe historique et naturel, fait d'espace et d'authenticité, la perception d’un territoire singulier qui m’ont donné la mesure de la complexité des regards à expliciter. Il faut accepter de changer de paradigme, de la simplification à la complexification, de la séparation à la conjonction (Morin 1977, 1980), de passer de celui du cristal à celui du fluide, des nuages, de la flamme ou de la fumée (Serres 1980), accepter de faire une sociolinguistique des réseaux, du lien, de la mouvance, de l'hétérogène (Heller 2002).

Et de là, par ce fil de la réflexivité qui caractérise lalterlinguistique (De Robillard 2008), il devient incontournable de poser non seulement ce qu'est pour nous la recherche mais aussi comment doit se faire la recherche qui nous intéresse. Au-delà de nos démarches de découvertes, de l'hypothico-déductif positiviste à l'empirico-inductif réflexif de notre sociolinguistique (Blanchet 2000), elle ne peut, à mon sens, se poser en pratique de déconstruction d'une technolinguistique mais dans une complémentarité ouverte et attentive, possible et utile, dans la mesure où il nous faut tenter parfois dadapter les outils de compréhension à ce que nous observons. La force d’une tel regard est d’affirmer les limites de lobjectivité mais en faisant, agissant, interprétant, tout de même et malgré tout, avec les moyens à notre disposition et dans la conscience de notre incomplétude. Parce que nous faisons de la recherche, nous nous devons de donner notre interprétation des phénomènes, 
interprétation subjective certes, et donc complexe et multiple mais, en tout cas, vraisemblable et argumentée.

Les phénomènes que j’ai choisi d'observer me semblent, comme l'envisage tout un chacun pris dans son activité de recherche, dignes d’intérêt. Sinon comment se sentir portée par la journée qui s'annonce ? On simagine alors que nos objets sont «stimulants intellectuellement ", sont propices à des théorisations nouvelles, ont été peu exploités ou répondent à des nécessités politiques et institutionnelles de carrière. Bref, que nous y trouvons du grain à moudre. Mais il me faut davantage encore ; il me faut trouver ma propre nécessité, comme si la compréhension d’un fragment du réel que je circonscris, participait de ma complétude, comme si javais étrangement besoin d'être nourrie par une compréhension et une narration pour affronter l'absurdité de la fin et ma propre béance, comme si en construisant du sens, je me donnais du sens, au moins dans l'espace de cette activité mentale. Tel Sisyphe croyant défier la mort mais finalement condamné à porter éternellement sa pierre au haut de la colline. Je m’égare. Je choisis done mes objets de recherche parce quills font écho à certaines de mes préoccupations sociales...et personnelles. Je sais qu’à travers mes différents projets et terrains, je cherche au fond à comprendre comment se manifestent, dans et par le langage, les liens diidentité et les changements en jeu dans les rapports sociaux. Je ne cherche pas seulement les langues à travers les «locuteurs", mais les discours et les interactions, faiseurs didentité, qui peuvent expliciter les processus sociaux, d’en comprendre les mécanismes, les rapports diintérêt de pouvoir et de domination, les liens, la mouvance, les imbrications, avec quelques outils de description. Mais comment alors analyser et me représenter ce que jobserve ? Au fil du temps, jai choisi des outils pour accomplir ma tâche, des outils à chaque fois appropriés à mes questions et mes données. Un outil est « doté d'une forme et de propriétés physiques adaptées à un procès de production déterminé qui permet de transformer l'objet de travail selon un but fixé. Au sens figuré, c’est ce qui permet d'obtenir un résultat, d'agir sur quelque chose (Trésor de la langue française 2009)". Dans mon apprentissage et d’objet en objet, j’ai donc sélectionné mes outils, ceux qui effectivement me semblaient les mieux adaptés aux données choisies et à mes fins de compréhension/interprétation, de façonnage et de confection. J'ai le sentiment quil m’a fallu toutes ces années pour savoir quels outils étaient vraiment les miens, pour «me les faire ", comme un bon facom « se fait » à la main tandis que le cal de la peau «se fait" lui aussi à l'outil. Je veux dire que je sais aujourd'hui que certains outils me sont indispensables, quils me conviennent, que je les ai vraiment en main, quils sont efficaces, 
fiables et solides. Ils vont servir mes découvertes et réalisations et je sais désormais, quand le besoin est là, aller chercher celui qui, adapté, affinera la pièce, celui qu’on avait oublié un instant mais qui accomplira les ultimes fabrication et exaucement (de l'analyse des tours de parole à quelque champ lexical ou à des marques pragmatiques et énonciatives). Dévoiler les mécanismes souterrains à notre propre analyse (nos choix de terrains multi-sites, leur délimitation, notre place de chercheur, nos intérêts personnels, nos émotions et représentations) enrichit aussi, dans un balancement de l'objectif au subjectif, linterprétation des données qui remontent du réel en langues, observables et identifiables (prise de parole ou pas, alternance ou pas, marqueurs de variation, croisement multimodal etc).

Cette recherche alterlinguistique sensible, intuitive et expérimentale, intègre trois phases (non pas dans la linéarité mais dans l'enchevêtrement), celle de la présence et de liimprégnation (du « terrain »), celle de la compréhension ( «analyse » serait plus surplombant ?) et celle centrale de l'écriture (De Robillard 2007). Notre compréhension/traduction/interprétation des situations relève donc à fois de l'expérience et de l'écriture. La façon de représenter, de sélectionner, de mettre en écriture, en métaphores parfois, est aussi déterminante (importante) que ce que l'on analyse. L'activité globale de décentrement devient un mode de saisie scientifique de la complexité. Il s’agit de faire de la « linguistique autrement ».

\section{Une épistémologie de la sociolinguistique}

Il deviendrait alors nécessaire, parce que nous sommes en manque de retour sur pratiques, de poursuivre et intensifier notre propre histoire, celle d'une sociolinguistique en France. Si elle a été entamée par quelques-uns de notre discipline, membres du Réseau Francophone de Sociolinguistique (entre autres, Blanchet et De Robillard (Eds) 2003, Blanchet, Calvet et De Robillard 2007, Calvet I993, I999, Gadet I97I, 2005), une «Histoire des idées sociolinguistiques" en trois tomes $(-)$, ouvrage qui dirait une façon de raconter cette sociolinguistique, ferait figure d’un geste inaugural. Il en irait, car nous sommes-là terriblement en retard, d’une histoire au sens strict, "épistémologie » au sens français, à une sociologie de la science donc de nos pratiques, institutionnelles et sociales (Bourdieu 200I, Braunstein 2008).

On verrait alors combien, en France, la sociolinguistique a été grandement occultée dans les années 70, malgré les jalons diglossiques et laboviens, par la mainmise du structuralisme, qui, vent en poupe, se faisait l'égérie des sciences humaines, dans une construction idéalisée et sans 
cesse réinterprétée du mythe Saussurien (Puech 2008). On verrait combien la linguistique simposait à travers son institutionnalisation, ses revues (Chevalier I998), elle qui jouait d'un positivisme entendu et des idéologies nationales autour d’une langue homogène (De Robillard 2008). Dans un paysage peu propice au doute, à la réflexivité et au mélange, la sociolinguistique n’a pas encore élaboré son histoire française d’une science du langage où le fait social serait sur le devant de la scène, avec Antoine Meillet, et son discipline Marcel Cohen, ou Charles Bally comme figures emblématiques du sujet (Chiss et Puech 1997, I999) quand Saussure ne serait plus le mythe de référence. Elle retracerait les échees de son ethnologie, du naturalisme linguistique (Desmet 1996, Auroux (Ed) 2007) au marrisme (Bertrand 2005), les difficultés de son implantation parce que jugée trop récente ou trop « incohérente " (Todorov 1972 : 84), le manque (hélas) de lien historique avec l'anthropologie linguistique et l'ethnographie interactionnelle américaines (Calvet I999), les tensions et courants internes à la discipline, et sinventerait un avenir (Ammon, Mattheier et Nelde 2000) mêlé aux réflexions européennes et nord-américaines déjà bien plus avancées.

\section{L’épreuve de la réflexivité}

Mais la réflexivité n’est pas de tout repos. Elle nous pousse à être sans cesse en équilibre et en doute quand rien n'est jamais fixé ni acquis.

\section{Le désordre et la perte de contrôle}

La question de l'ordre est centrale dans la vision (et son acceptation) positiviste du monde. Si la nature semble ne pas supporter le désordre (Morin I977, I980) et cherche toujours un équilibre perdu, l'homme est dans une quête réconfortante, mais inépuisable et impossible, de classement, catégorisation pour justement mettre de l'ordre, trouver de l'ordre, affirmer l'ordre. Il s'agit sans cesse de contrôler, maîtriser la perte, l'échappée, lỉnsaisissable (Elias I987) qui renvoient, à la fin absurde de sa propre finitude ; comme sill fallait contrer la vanité (insignifiance) de sa vie. L'ordre rassurant est contraire à la perte de soi.

La perte de soi. Lindividualisme consacre aujourd'hui le sujet dans toute sa subjectivité ; il reconnaît l'acteur social faiseur de sa propre vie, de son «individuation ", et fort de ses désirs personnels - pour un épanouissement intime et collectif -, et donc pour le meilleur. On ne peut pourtant nier, combien face au désir et à la volonté de construire son propre destin individuel, la participation à la postmodernité entraîne aussi désespérance, fragilités et «fatigue d'être soi » (Ehrenberg 1998). Lindividualisme est pris dans cette dualité impossible, création de soi 
et libre initiative, et désespérance de ne pouvoir répondre à cette injonction de grande liberté. Quand ni la morale, ni l’ordre historique et social nïmposent les conduites de vie, lindividu contraint par son libre-arbitre, peut se perdre dans la désassurance et la fragilité. Dans une société de la prise de risque, seul lindividu se voit responsable de son destin, de ses réussites mais aussi de ses échecs. L'activité réflexive, pilier complémentaire de l'action dans la construction d’identité (Kaufman 2004), peut introduire le doute et la fragilité, contre toute certitude solidement ferraillée. Il en est de même dans l'activité réflexive du chercheur. L'activité de recherche réflexive peut introduire quête et doute de soi, dans un parcours de déconstruction, dans le risque de ne pas se trouver, de ne pas être compris dans ses chemins de traverse. Mais dans ces moments, je sais qu'il me faut lire celles dont les pensées en liberté me sont proches, celles (des chercheures en proximité) qui veulent " penser ailleurs » (Thomas I998, Lapierre 2004), et toujours oser entrer en conversation (Heller 2002).

\section{Le franchissement des frontières}

Pourtant l'ordre aujourd'hui, corseté dans des frontières disciplinaires, se craquelle; les réponses à nos questions, pour être satisfaisantes, flirtent avec d’autres approches et disciplines, la littérature, la poésie et les autres sciences sociales. Sans oublier quill y a de la jubilation intellectuelle voire créatrice à aller chercher dans la littérature, le cinéma ou la chanson des points de ralliements, des complicités et des éclairages, comme jaime à le faire. «Je suis enchanté de trouver des parallélismes dans des domaines qui me paraissent les plus étrangers, les plus éloignés les uns des autres. [...] Il y a donc un plaisir d'écrivain à sortir des sentiers battus en faisant circuler une courbe à travers des points de repère complètement hétérogènes et dont je m’attache à montrer la grande homogénéité (Rosset 200I)».

J'ai eu besoin de la géographie sociale, de l'ethnologie urbaine, de l'histoire pour saisir quelques éléments explicatifs de mes recherches. La géographie sociale me pousse à penser dans une troisième dimension, quand l'homme n'est plus seulement un sujet de parole mais d’espace, quand la représentation du monde se fait aussi par le dessin, le schéma, le croquis, alors que mes propres représentations, mes mises en compréhension et en sens se font par de la linéarité, du récit et du raconté. Les visions se croisent, s’alimentent, se forgent les unes les autre, quand la représentation des objets de l'espace de se fait plus plus dans la dimension cartésienne de celle d'objets isolés, d’un monde de cristal (Serres I980, lu suite à une riche conversation avec Bernard Debarbieux à Marrakech). Cette représentation de cristal est celle 
de la linguistique à laquelle se cogne la sociolinguistique, comme la géographie sociale à la géographie. Ces croisements en conversations avec l'histoire ou avec l'ethnologie revisitent nos modes de recherche, notre histoire disciplinaire au sein des sciences humaines, notre perception d'un réel flou, aléatoire, complexe. Surveiller les frontières de ses propres disciplines, rester dans leurs limites rassurantes serait encore une façon de faire de l'ordre mais aussi de chérir son savoir et ses prérogatives dans un effet de pouvoir et de distinction et dans une plus grande reconnaissance académique.

\section{Les limites du décentrement}

Pour autant, notre activité réflexive (loin d’une introspection qui nous mènerait à l'autobiographie ou à l'autofiction) a pour objectif, ce qui fait la recherche et ce pour quoi nous sommes rémunérés, un questionnement sur le monde social et linguistique. C’est là finalement le travail, que nous ne devons lâcher, même sill reste particulièrement acrobatique et que nous en connaissons les limites méthodologiques: représentativité des données, temps de la recherche, accès aux situations. La réflexivité polarise ces difficultés pratiques, cet embarras parfois à saisir l’altérité, mais nous induit de faire avec, et malgré tout, loin de tout relativisme absolu. On sait quau nom de la nécessaire déconstruction, certains anthropologues américains ont complètement cessé de faire du terrain (pour lutter d’ailleurs contre la domination occidentale du savoir et le regard surplombant). "L'anthropologie s'est complètement transformée en une textologie critique, en une traque de toutes les culpabilités ethnocentriques (Godelier 2004: 196)». Il faut rester vigilant, pour que la réflexivité soit un vecteur permanent du doute fondamental mais non une mainmise de lintime (parce que ce au fond pas si intéressant que ça pour la recherche) ou une forme de déconstruction paralysante et improductive.

\section{La réflexivité construite dans linteraction}

La réflexivité est singulière en sociolinguistique dans le sens où qu'elle passe par la parole en interaction avec autrui, de celle-là même qui est notre objet d’étude. Le processus réflexif se situe en partie dans une parole, dont celle du chercheur, qui se révèle dans la relation à l'autre. Parce que nous savons que le langage n'est pas transparent, mais quil est polyphonique, implicite et hétérogène, linntersubjectivité, en œuvre dans toute interaction, est partie prenante des analyses, particulièrement quand le chercheur est embarqué sur le terrain. Dans une 
nécessaire décentration, la considération des catégories de l'autre, qui détermine notre interprétation du réel, passe par les discours et interactions mobilisés dans nos enquêtes où nous sommes nous-mêmes acteurs. Ainsi, le sujet, et donc nous-même, se construit en discours et en parole, avec l'autre; la rencontre de subjectivités est à aller chercher dans les pratiques langagières que nous provoquons ou saisissons (pourquoi celles-là et pas d'autres, pourquoi cette question, pourquoi ces représentations ?).

Mais limpasse est latente quand le sujet n'est jamais donné, quill ne peut être que dans une illusion de lui-même, d’un moiqui se voudrait construction sûre et figée mais trompeuse, d’une certitude diidentité homogène qui se délite pourtant dans les failles de lintersubjectivité ; liidentité du sujet en je, rompue à lincomplétude, est prise dans la perception de l'autre, de son retour sur image entre liens et distances. De ces écheveaux et fils tissés, se vivent des identités plurielles constitutives d'hétérogène, pourtant actualisées en situation et en langues au-delà d’un moi, sûr et posé quỉl semble donner le plus souvent à voir. Donc le sujet naît d’un autre, révélateur de différence dans une émergence de parole. Cet autre par sa présence effective en interaction donne à se dire au sujet qui s’échappe à lui-même à travers boucles réflexives, lapsus, marques énonciatives, reformulations, représentations, affirmations, circulations de discours etc. "Le langage serait alors davantage une activité envers l'autre, une pratique d'altérité voire d'aliénation, qui serait en termes positifs l'autre dans sa dynamique et non pas dans sa neutralité (Siouffi 200I: I85)». Jusque dans les conversations ordinaires, cette complexité du sujet est à expliciter pour que nos questions de recherche trouvent sens et significations.

\section{Pour finir}

Allons-y alors, même si notre activité d’analyse sociolinguistique est prise par la contrainte de son « objet », la langue comme activité de parole. Je me suis toujours sentie à la fois enserrée et libre dans notre outil, la langue pour raconter. Enserrée parce que prise avec la linéarité pour parler des phénomènes, et des évènements dans leur globalité, d’où pour moi cette nécessité à un moment d'aller voir du côté de la danse et du corps, dans une autre forme de contrôle social. Mes idées et mes explications se construisent en faisceau ; la parole me bride parfois ; jaime la peinture pour sa saisie globale, sa traduction du monde dans une essence totale, la poésie créole d'un monde de langues mêlées (Glissant 2009); juse des métaphores à l'écrit pour leur évocation et leur subtilité dans l'interprétation/traduction. 
Allons-y et trouvons nos propres mises en ouvre. Expérimentons, tentons et osons une sociolinguistique, qui serait en France dans la pleine reconnaissance d’elle-même.

\section{Références bibliographiques}

Abélès M., 2004, "Le terrain et le sous-terrain", Ghasarain C. (Ed), De l'ethnographie à l'anthropologie réflexive, Paris, Armand Colin : 35-40

Ammon U., Mattheier K. et Nelde P., 2000, Die Zukunft der europäischen Soziolinguistik, The Future of European Sociolinguistics, Le futur de la sociolinguistique européenne, Revue Sociolinguistica, Tübingen, Max Niemeyer Verlag

Auroux S. (Ed), 2007, Le naturalisme linguistique et ses désordres, Revue Histoire, épistémologie, Langage, Tome XXIX, fascicule 2, Paris, Université Paris 7 Bachelard G., I938, La psychanalyse du feu, Paris, Gallimard

Bertrand F., 2005, «N. Marr et le marrisme pour l'ethnographie soviétique des années I920I930 ", Cahiers de l'ILSL, numéro $20: 27-38$

Blanchet P. 2000, Linguistique de terrain, méthode et théorie. Une approche ethnosociolinguistique, Presses universitaires de Rennes

Blanchet P. et De Robillard (Eds), 2003, Langues, contacts, complexité. Perspectives théoriques en sociolinguistique, Presses universitaires de Rennes

Blanchet P., Calvet L.-J., De Robillard D. , 2007, Un siècle après le Cours de Saussure : la Linguistique en question, Carnets d'Atelier de Sociolinguistique, L'Harmattan

Braunstein J.-F. 2008, "Désunités, styles et épistémologies historiques", Histoire des sciences. Méthodes, styles et controverses, Paris, Vrin : 227-224

Bourdieu P., 200I, Science de la science et réflexivité, Paris, Raisons d’agir

Calvet L.J., I993, La sociolinguistique, Paris, Puf, Que sais-je ?

Calvet L.J, I999, "Aux origines de la sociolinguistique. La conférence sociolinguistique de l'UCLA », Langage et société, numéro 88 : 25-57

Chevalier J.-L., I998, « Place des revues dans la constitution d'une discipline : la linguistique française (I947-I997), Langue française, volume II7, numéro I : 68-8I

Chiss J.-L. et Puech C., I997, Fondations de la linguistiques. Etudes d'histoire et d'épistémologie, Paris, Duculot

Chiss J.-L. et Puech C., I999, Le langage et ses disciplines, Paris, Duculot 
Debarbieux B., 2008, «Le syndrome de Moctezuma ou réflexions sur l'actualité et la pertinence du couple ville-campagne dans l'analyse territoriale ", Michèle Tranda M. , Lilli Monteventi Weber L., Chantal Deschenaux C. (Eds), Campagne-ville : le pas de deux: Enjeux et opportunités des recompositions territoriales, Paris, presses polytechniques : 6I-75

De Robillard 2007, «La linguistique autrement: altérité, réflexivité, constructivisme, multiversalité : en attendant que le Titanic ne coule pas", Un siècle après le Cours de Saussure : la Linguistique en question, Carnets d'Atelier de Sociolinguistique, L'Harmattan : $8 \mathrm{I}-228$

De Robillard D., 2008, Perspectives alterlinguistiques, Espaces discursifs, L'Harmattan Desmet P., 1996, La linguistique naturaliste en France (I867-1922): nature, origine et évolution du langage, Louvain, Peeters Publishers

Ehrenberg A., I998, La fatigue d’être soi, Paris, Odile Jacob

Elias N., I987, La solitude des mourants, Christian Bourgois

Gadet F., I97I, "Recherches récentes sur les variations sociales de la langue ", Langue française, Volume 9, numéro I : 74-8I

Gadet F., 2005, "I977 : sur un moment clé de l'émergence de la sociolinguistique en France », Cahiers de l'ILSL, numéro 20 : I27-I38

Glissant E. et Chamoiseau P., 2009, Lïntraitable beauté du monde Adresse à Barack Obama, Paris, Galaade

Glissant E., 2009, Philosophie de la relation. Poésie en étendue. Paris, Gallimard.

Godelier M., 2004, "Briser le miroir du soi", Ghasarain C. (Ed), De l’ethnographie à lanthropologie réflexive, Paris, Armand Colin : 193-2IO

Heller M., 2000, «Pouvoir et frontières sociales dans l'exercice de la recherche", dans Forschungsethik und Minderheiten, Grenzgänge, n ${ }^{\circ}$ 33 : 9-I5

Heller M., 2002, Eléments d’une sociolinguistique critique, Paris, Didier

Kaufman J.-C., 2004, Lïnvention de soi. Une théorie de lidentité. Armand Colin

Lapierre N., 2004, Pensons ailleurs, Paris, Stock

Moïse C., 2004, (2004) «Le Nouvel Ontario, nordicité et identité », Heller M. et N. Labrie (Éds), Discours et identités. Le Canada français, entre modernité et mondialisation, chapitre 2, Editions modulaires européennes, Fernelmont, Belgique : 43-88 
Moïse C., 2007, « Déambulations sociolinguistiques », Lambert P., Millet A., Rispail M. et Trimaille C. (Éds), Variations au coeur et aux marges de la sociolinguistique. Mélanges offerts à Jacqueline Billiez, Paris, L'Harmattan : I79-I86

Moïse C., Budach G., et Kahn E., 2009, «Un projet transnational francophone : méthode d’analyse pour appréhender valeurs et idéologies dans la définition d’un Canada français », Langues, identités et savoirs en milieu minoritaire à l'heure de la mondialisation, Langage et Société

Morin E, I977, La méthode. La Nature de la Nature, Paris, Points Essais

Morin E., I980, La méthode. La vie de la vie, Paris, Points Essais

Puech C., 2008, «Qu’est-ce que faire l'histoire du récent ? ", Congrès Mondial de

Linguistique, http://dx.doi.org/IO.IO5I/cmlfo8334

Prieur J.-M., 200I, "Qui ? ”, Langues en contact et incidences subjectives, Revue Traverses,

Presses de l’université Montpellier 3, numéro 2 : II-2I

Rosset C., 200I, «Entretien », 2 I penseurs pour comprendre le XXIe siècle, Le Monde de

l'éducation : 26-32

Serres M., I980, Hermes V, La passage du Nord-Ouest, Paris, Minuit

Siouffi G., 200I, «Identité, aliénation, langage », Subjectivités, singularités, cultures, revue Traverses, numéro 3, Presses de l’université Montpellier 3 : I8I-I97

Todorov Z., I972, "Sociolinguistique», Ducrot O. et Todorov T., I972, Dictionnaire encyclopédique des sciences du langage, Paris, Seuil : 83-9I

Thomas C., I998, Comment supporter sa liberté ?'Rivages Poche

Todorov Z., I989, Nous est les autres, Paris, Seuil 